\title{
Pierídeos (Lepidoptera: Pieridae) da Coleção de Invertebrados do Instituto Nacional de Pesquisas da Amazônia, INPA
}

Karla Christiane Ayden LEITE ${ }^{1}$, Catarina da Silva MOTTA ${ }^{1}$ Francisco F. XAVIER-FILHO${ }^{1}$

RESUMO

As espécies de Pieridae da Coleção de Invertebrados do Instituto Nacional de Pesquisas da Amazônia foram inventariadas. São registrados 279 indivíduos de 17 espécies e 10 gêneros, todos coletados na Amazônia brasileira.

PALAVRAS-CHAVE

Lepidoptera, Pieridae, coleção sistemática, Amazônia brasileira

\section{Pierid butterflies (Lepidoptera: Pieridae) of the Instituto Nacional de Pesquisas da Amazônia, INPA}

\section{ABSTRACT}

The species of Pieridae of the collection of Invertebrates of the National Institute for Amazonian Research were inventoried. It was registered 279 individuals of 17 species and 10 genera, all collected in the Brazilian Amazonia.

KEYWORDS

Lepidoptera, Pieridae, Systematic collection, Brazilian Amazonia

1 Instituto Nacional de Pesquisas da Amazônia, INPA, Coordenação de Pesquisas em Entomologia (CPEN), Av. André Araújo, 2936, Bairro Petrópolis, C.P. 478, 69011-970, Manaus, AM. e-mail: motta@inpa.gov.br 
A família Pieridae se divide em quatro subfamílias: Dismorphiinae, Coliadinae, Pierinae, e Pseudopontiinae (esta última com apenas uma espécie ocorrendo na África) (De Vries, 1987) e forma um grupo de borboletas com grande diversidade de espécies (cerca de 1.200 spp.) com ocorrência em todos os continentes, exceto na Antártida, sendo mais bem representados nos trópicos (Scoble, 1995). No Neotrópico ocorrem 323 espécies de Pieridae e no Brasil 65 espécies (Brown Jr., 1996). Mais recentemente este número foi elevado para 339 espécies no Neotrópico (Lamas, 2004). Os pierídeos têm grande diversidade de cores, mas a maioria tem como cores predominantes o amarelo, alaranjado ou branco. Algumas espécies são consideradas pragas agrícolas, pois suas lagartas alimentam-se de culturas economicamente importantes, especialmente crucíferas (DeVries, 1987). Os adultos se alimentam principalmente de néctar em flores e grandes bandos (paná-paná) freqüentemente se formam para se alimentar de sais em areia úmida, em poças e praias dos rios.

Este trabalho inventaria as espécies depositadas na Coleção de Invertebrados do Instituto Nacional de Pesquisa da Amazônia, INPA, coletados nos estados do Amazonas, Pará, Roraima, Rondônia e Mato Grosso. No Amazonas especialmente nos municípios de Carauari (05006’31"S / 67010'30”W) em junho de 2005; Presidente Figueiredo,
BR-174, km 113 (02²8'56"S / 6001'48”W) em julho de

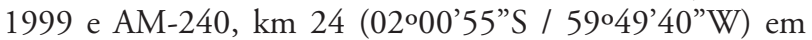
maio e setembro de 2005; Manaus (030.5'45"S/59059'20”W) desde 1970 até 2005. Os espécimes foram identificados por comparação direta com exemplares da coleção do INPA ou por comparação com figuras e/ou fotos de literatura especializada (Lewis, 1975; D’Abrera, 1981; Mielke \& Casagrande, 1991). A nomenclatura foi atualizada de acordo com Lamas (2004). Em todos os espécimes foram acrescentados etiquetas com número de Registro do INPA e os dados das etiquetas foram colocados em Livro de Tombo e incorporados ao banco de dados da Coleção.

Foram registrados 279 exemplares de 17 espécies em 10 gêneros. Os machos somaram 91\% dos espécimes (Tabela $1)$.

Apesar dos pierídeos serem comuns, a Coleção do INPA possui um número baixo, $(26.1 \%$ das espécies da fauna brasileira) quando comparado, por exemplo, com as coletas realizadas em um único local, na Estação Científica Ferreira Penna, na Floresta Nacional de Caxiuanã onde foram registradas 21 espécies em 11 gêneros, equivalendo a 32,3\% dos pierídeos do Brasil (Overal \& Silva, 2002). Fica evidente a necessidade de um esforço de coleta maior para se obter uma boa representatividade da fauna da região.

Tabela 1 - Pierídeos da Coleção de Invertebrados do Instituto Nacional de Pesquisas da Amazônia

\begin{tabular}{|c|c|c|c|c|}
\hline Subfamílias & Espécies & Machos & Fêmeas & Total \\
\hline \multirow[t]{6}{*}{ Pierinae } & Glutophrissa d. drusilla (Cramer, 1777) & 23 & 02 & 25 \\
\hline & Itaballia demophile (Linnaeus, 1763) & 06 & 02 & 08 \\
\hline & Melete I. Iycimnia (Cramer, 1777) & 06 & & 06 \\
\hline & Perrhybris p. pamela (Stoll, 1780) & 03 & & 03 \\
\hline & Ascia monuste orseis (Godart, 1819) & 11 & 02 & 13 \\
\hline & Hesperocharis nera (Hewitson, 1852) & 01 & & 01 \\
\hline \multirow[t]{11}{*}{ Coliadinae } & Anteos menippe (Hübner, [1818]) & 51 & 01 & 52 \\
\hline & Phoebis a. argante (Fabricius, 1775) & 21 & 01 & 22 \\
\hline & Phoebis s. sennae (Linnaeus, 1758) & 04 & 03 & 07 \\
\hline & Phoebis p. philea (Linnaeus, 1763) & 06 & 01 & 07 \\
\hline & Rhabdodryas trite (Linnaeus, 1758) & 08 & & 08 \\
\hline & Phoebis agarithe (Boisduval, 1836) & 01 & & 01 \\
\hline & Aphrissa s. statira (Cramer, 1777) & 66 & 06 & 72 \\
\hline & Pyrisitia n. nise (Cramer, 1775) & 16 & & 16 \\
\hline & Eurema (Eurema) e. elathea (Cramer, 1777) & 06 & 05 & 11 \\
\hline & Eurema (Eurema) p. phiale (Cramer, 1775) & 04 & 01 & 05 \\
\hline & Eurema (Eurema) albula (Cramer, 1775) & 21 & 01 & 22 \\
\hline Total & & 254 & 25 & 279 \\
\hline
\end{tabular}




\section{AGRADECIMENTOS}

Os autores agradecem ao Dr. Augusto Loureiro Henriques pelos comentários valiosos. Ao Dr. Olaf Hermann Hendrik Mielke que gentilmente nos enviou cópia do trabalho de Overal \& Silva (2002).

\section{BIBLIOGRAFIA CITADA}

Brown Jr., K.S. 1996. Diversity of Brazilian Lepidoptera: history of study, methods for measurement, and use as indicator for genetic, specific and system richness. In: C.E.M. Bicudo \& N.A Menezes (eds.). Biodiversity in Brazil: a first Approach. São Paulo CNPq, p. 221-253.

D'Abrera, B. 1981. Butterflies of the Neotropical Region. Part 1 Papilionidae \& Pieridae. Oxon, Lansdowne \& Classey ed. 172 pp.

De Vries, P. 1987. The Butterflies of Costa Rica and their natural history: Papilionidae, Pieridae, and Nymphalidae. New Jersey: Princeton Univ. Press. 327pp.
Lamas, G. 2004. Checklist: Part 4A, Hesperioidea - Papilionoidea, (Lamas ed.). In: J.B. Heppner (ed.). Atlas of Neotropical Lepidoptera. Gainesville, Association for Tropical Lepidoptera, Scientific Publishers. 439pp.

Lewis, H.L. 1975. Las Mariposas del Mundo. Barcelona: Ed. Omega. $312 \mathrm{pp}$.

Mielke, O.H.H.; Casagrande, M.M. 1991. Lepidoptera: Papilionoidea e Hesperioidea coletados na Ilha de Maracá, Alto Alegre, Roraima como parte do Projeto Maracá, com uma lista complementar de Hesperiidae de Roraima. Acta Amazonica, 21: 175-210.

Overal, W.L.; Silva, P.J.B. 2002. Borboletas, In: Lisboa, P.L.B. (org.) Caxiuanã: Populaçôes tradicionais, Meio Físico e Diversidade Biológica, Belém: Museu Paraense Emílio Goeldi. p. 521-532.

Scoble, M.J. 1995. The Lepidoptera: Form, Function and Diversity. The Natural History Museum, London \& Oxford University Press. 440pp.

Recebido em 06/10/2005

Aceito em 29/03/2007 